Research Article

\title{
Application of Deep Learning in Financial Management Evaluation
}

\author{
Wenlei Shi, ${ }^{1}$ Lei Xu $\mathbb{D}{ }^{2}$ and Dongli Peng ${ }^{3}$ \\ ${ }^{1}$ School of Accounting, Shandong University of Finance and Economics, Jinan 250014, Shandong, China \\ ${ }^{2}$ School of Economics and Management, Qilu Normal University, Jinan 250000, Shandong, China \\ ${ }^{3}$ School of Public Administration, Hengshui University, Hengshui 053000, Hebei, China \\ Correspondence should be addressed to Lei Xu; xulei@qlnu.edu.cn
}

Received 24 September 2021; Revised 12 October 2021; Accepted 13 October 2021; Published 3 November 2021

Academic Editor: Tongguang $\mathrm{Ni}$

Copyright (C) 2021 Wenlei Shi et al. This is an open access article distributed under the Creative Commons Attribution License, which permits unrestricted use, distribution, and reproduction in any medium, provided the original work is properly cited.

The competition among enterprises is becoming increasingly fierce. The research on the financial management evaluation model is helpful for enterprises to find possible risks as soon as possible. This paper constructs the financial management evaluation model based on the deep belief network and applies the analytic hierarchy process to determine the weight of financial management evaluation indicators, which is compared with other classical deep learning evaluation methods, such as KNN, SVM$\mathrm{RBF}$, and SVM linear. It has achieved an accuracy of more than $81 \%$, showing a satisfactory prediction effect, which is of great significance to formulate corresponding countermeasures, strengthen financial management, improve the capital market system, and promote high-quality economic development. In addition, aiming at the problem of abnormal financial data, this paper uses the new sample dataset obtained by principal component analysis for convolution neural network model learning, which enhances the prediction accuracy of the model and fully shows that deep learning is feasible in the research of financial management prediction, and there is still a lot of space to explore.

\section{Introduction}

With the development of society, financial intelligence increasingly affects our life and has a great impact on the traditional financial work, which is a topic of concern to enterprises all over the world. By importing data into the database or taking the existing data in the database as the analysis object [1], financial intelligence processes the data according to the financial management model and uses the high-speed and accurate computing power of the computer to quickly obtain the enterprise operation diagnosis report, so as to form a fast and reliable basis for business decisionmaking [2]. As a popular direction in the computer field, deep learning technology has been closely combined and applied with the financial field. Using reasonable deep learning technology can solve the problem of efficient automatic data analysis in the financial industry, provide valuable prediction information for managers, and provide reliable early warning for healthy institutional operation [3].
Throughout the research trends of financial management evaluation at home and abroad, it mainly focuses on financial evaluation indicators and evaluation models. In the selection of financial management evaluation indicators, existing studies mainly focus on which indicators can accurately predict enterprise crisis [4]. It has experienced the common application stage of multidimensional indicators from single financial ratio indicators and multivariable financial ratio indicators to the combination of financial indicators and nonfinancial indicators [5]. The above studies have achieved certain prediction results, but the selection of multidimensional early warning indicators usually uses statistical methods to test the normality and significance of sample index data and then combined with manual discrimination based on professional ability to select indicators. The selection method is more complex, and there is no unified conclusion on which indicators to select. In the selection of early warning models, early scholars often used univariate model, logistic model, discriminant analysis 
model, and so on [6]. With the development of information technology, a large number of open real data information can be obtained in the process of enterprise operation. These data can often reflect the past risk status of the enterprise and even some characteristics of the risk of the whole capital market [7]. Therefore, scholars began to apply neural network, support vector machine and other models based on artificial intelligence methods to financial crisis early warning. Generally speaking, the early warning model based on artificial intelligence method has made great progress in the mechanism and systematicness of early warning. It not only overcomes the limitations of the early warning model based on statistical method, such as requiring data to obey normal distribution and complex calculation and analysis, but also has strong fault tolerance and learning ability [8]. At present, artificial neural network has developed to the stage of deep learning network, which is characterized by selflearning and high dynamic adaptability. Therefore, deep learning is also applied to the field of financial management evaluation.

Deep learning can complete a lot of regular, simple, and repetitive work. The application of financial intelligence technology in the financial field has played an important role in improving business efficiency, reducing work errors, and preventing and controlling enterprise risks. This paper studies the financial risk by establishing the financial management evaluation model, which is a quantitative method. It is different from the qualitative analysis. The work is difficult, and the accuracy is low. The model research has higher reliability and uses the analytic hierarchy process to determine the enterprise evaluation index system. This model interprets the historical data, links the characteristics of the data with the financial situation of the enterprise, and then uses the existing data to analyze and predict the future, so as to ensure the accuracy of the prediction.

\section{Related Work}

$\mathrm{Ng}$ et al. proposed a new fuzzy CMAC (cerebellar model articulation controller) model based on reasoning component rules as a new method for bank fault classification and early warning system [9]. Artificial neural network is introduced into financial management evaluation for the first time. Artificial neural networks not only deal with the lack of data errors, but also allow for timely adjustment of internal control parameters. Erhan et al. have proved through practice that the unsupervised training method can better describe the complex functional relationship, which provides a good reference for financial risk early warning. Through the establishment of Yahoo information bulletin board, Jones studied the impact of network information on financial management by using the method of deep learning $[10,11]$. Through the establishment of online information bulletin board, Jones reduced the fluctuation of stock price and found that investors' differences of opinion may reduce risk and increase turnover. Through the analysis of stock related blog information in support vector machine, Choudhury found that the fluctuation of stock price will be affected by blog content [12]. Tetlock conducted a 24-year analysis of 500 listed companies [13] and counted the negative words in various reports in this period through the method of deep learning. On the basis of the relationship between corporate income and stock income, this paper points out that the negative words in the news reports of listed companies can predict the decline of listed companies' profits and reevaluate the listed companies. Under the pressure of trading, news content may capture some information that is difficult to quantify, which has a certain early warning function in media reports. Najafabadi et al. abstracted the deep learning algorithm into data representation through the hierarchical learning process [14]. This method has attracted great attention from data science. It is widely used to solve problems, such as network security, medical information, national intelligence, and marketing. Jason Kuen and chin poo Lee applied deep learning to the representation of visual tracking invariance [15] and achieved good results through strong spatiotemporal constraints and stacking slow convolution tracking. Yudistira and Kurita [16] proposed that deep learning processor has become the most potential solution to accelerate deep learning algorithm and pointed out the disadvantage of low efficiency of assembly instructions written by deep learning, which puts forward higher requirements for the research of deep learning.

To sum up, although there are many researches on deep learning at home and abroad, covering a wide range of fields, they mainly focus on theory, speech recognition, and image recognition, which are the feature judgment of known information and lack of relevant empirical research. Many literature studies show that deep learning can well describe complex functions, and financial management evaluation can also be used as a complex function judgment, but so far, deep learning has not been used in the research of financial management evaluation. Based on the previous research, combined with the characteristics of financial management, this paper applies deep learning to the evaluation model of financial management.

\section{Evaluation Modelling Based on Deep Belief Network}

3.1. Deep Belief Network Model. Deep learning is a category of machine learning that focuses on neural networks. Machine learning can be applied to image, speech, pattern recognition, weather prediction, stock price prediction, gene expression, and content recommendation. This is very similar to the recognition of images by the cerebral cortex. The deep learning model first extracts the low-level features from the original signal, then obtains the higher-level features from the low-level features, and then obtains the higher-level expression. In the face recognition system, the original signal is the pixel, the low-level feature is the edge of the object, the high-level feature is the contour composed of edges, and the high-level expression is the face. Through training with characteristic data, the error is transmitted from top to bottom to fine tune the network. Based on the parameters of each layer obtained in the first step, the parameters of the whole multilayer model are further 
optimized. Finally, it classifies according to the high-level characteristics and outputs the prediction results of the model. Deep belief network is one of the mainstream deep learning algorithms [17]. According to the Boltzmann machine model of stochastic neural network, the principle architecture of restricted Boltzmann machine [18] is shown in Figure 1.

$a=\left(a_{1}, a_{2}, \ldots, a_{n_{v}}\right)^{T}$ represents the offset vector of the visible layer, $b=\left(b_{1}, b_{2}, \ldots, b_{n_{h}}\right)^{T}$ represents the offset vector of the hidden layer, and $W=\left(w_{i, j}\right) \in R^{n_{h} * n_{v}}$ is the weight matrix. The energy function in a deep confidence network generated by multiple constrained Boltzmann machines for any set of neurons with state vector $(\mathrm{v}, \mathrm{h})$ is expressed as follows:

$$
E(v, h \mid \theta)=-\left[\sum_{i=1}^{n_{v}} a_{i} v_{i}+\sum_{j=1}^{n_{h}} b_{j} h_{j}\right]-\sum_{i=1}^{n_{v}} \sum_{j=1}^{n_{h}} a_{i} b_{j} h_{j} v_{i}
$$

where $n_{v}$ is the number of all neurons, $v$ is the state vector, $h$ is the state vector in hidden layer, and $n_{h}$ is the number of all neurons in the hidden layer, and $\theta=\left\{a_{i}, b_{j}, w_{i, j}\right\}$ denotes the adjustment factors that limit the Boltzmann machine architecture. Through the energy function defined in formula $(1)$, we get the joint probability distribution of state $(v, h)$, as shown in

$$
P(v, h \mid \theta)=Z(\theta)^{-1} \exp [-E(v, h \mid \theta)],
$$

where the expression of $Z(\theta)$ is shown in

$$
Z(\theta)=\sum \exp [-E(v, h \mid \theta)],
$$

where $Z(\theta)$ is the normalization parameter. It can be seen that in order to obtain $p(v \mid \theta)$ and $p(h \mid \theta)$, the key step is to calculate the normalized parameter $Z(\theta)$. The connection of a DBN is guided and determined by generating weights from top to bottom. RBMs is like a building block. Compared with the traditional and deeply layered sigmoid belief network, it can easily learn the connection weights. The probability of activation of a neural unit in the hidden layer can be calculated by the following formula [19]:

$$
P\left(h_{j}=1 \mid v, \theta\right)=\sigma\left(b_{j}^{2}+\sum_{i} \sqrt{2} v_{i} w_{i, j}\right),
$$

where $\sigma(\bullet)$ indicates sigmoid activation function. The stochastic gradient algorithm is usually used to find the maximum value of $\sum_{i=1}^{t} \log P\left(v_{i} \mid \theta\right)$. The momentum coefficient is generally taken as $(0,1)$. Intuitively, it is understood that if the current gradient direction is the same as the gradient direction of the previous step, the weight update of this step will be increased, and if it is different, the update will be reduced. The depth belief network model adopted is shown in Figure 2.

3.2. Determination of Financial Management Evaluation Index by Analytic Hierarchy Process. The evaluation of financial management objectives of logistics enterprises is a complex systematic project, which requires the establishment

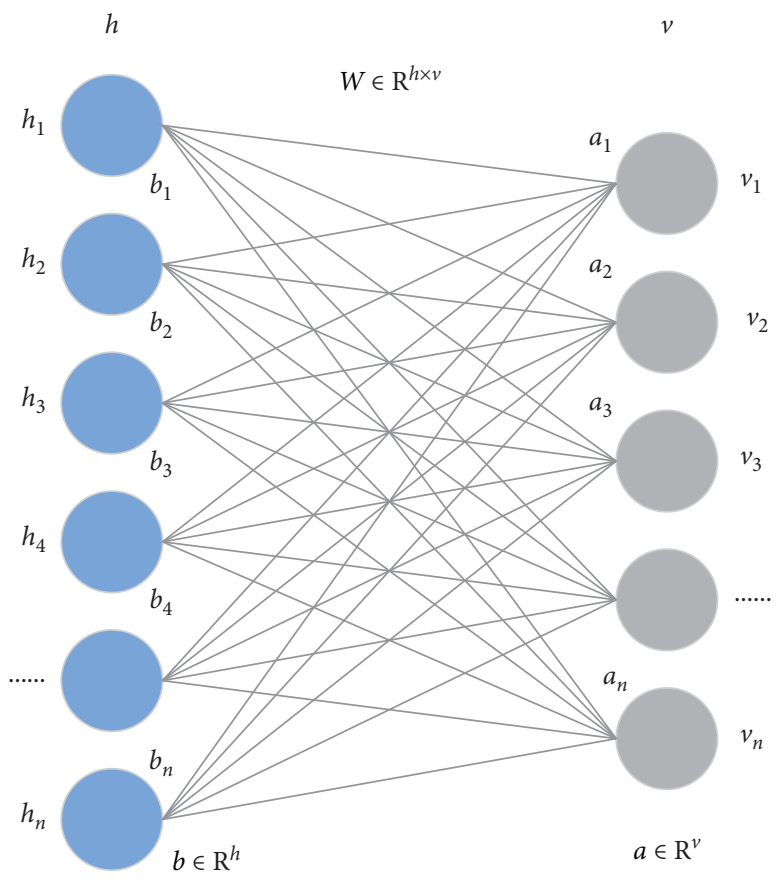

FIGURE 1: Restricted Boltzmann machine model.

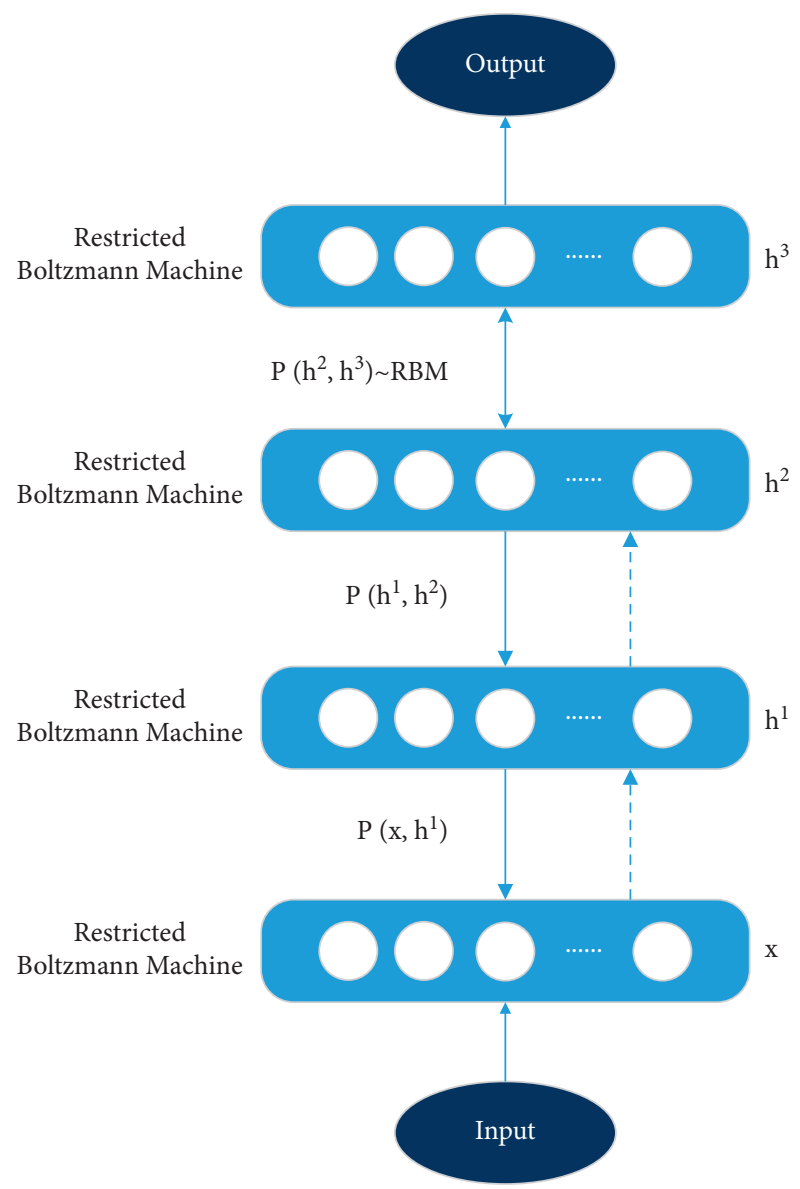

FIgURE 2: Deep generative model. 
of a financial management evaluation system [20]. ST means "special treatment." The policy is aimed at those with abnormal financial or other conditions. The addition of * ST before the stock means that the listed company has suffered losses for three consecutive years, and the exchange makes a delisting warning. The weight of financial management evaluation indicators is determined by analytic hierarchy process. Analytic hierarchy process decomposes the problem into different constituent factors and gathers and combines the factors according to different levels according to the correlation, influence and subordinate relationship between the factors to form a multilevel analysis structure model. It is a model and method for making decisions on complex systems that are difficult to be fully quantitative. The steps are as follows. First, establish a financial management evaluation index system. In order to make a correct evaluation of financial management, we should start from the corporate governance structure; establish the financial management evaluation system from the perspectives of management decision-making and external environment. The corporate governance structure, management decision-making and external environment evaluation are composed of some related elements. See Figure 3 for details.

Then, the judgment matrix $A$ is constructed to obtain the weights of $U 1, U 2$, and $U 3$ of the evaluation index system. As an example, the calculation process is illustrated. For the above evaluation index system, the expert group believes that in the evaluation of financial management, corporate governance structure $(U 1)$ is more important than management decision (U2) and external environment (U3), and the management decision is more important than the external environment; then $A=\left[\begin{array}{ccc}1 & 2 & 3 \\ 1 / 2 & 1 & 2 \\ 1 / 3 & 1 / 2 & 1\end{array}\right]$.

Calculate the product $M i$ of each row element of the judgment matrix, and then calculate the $n$th root of $M i$. Finally, normalize the vector $\left[W_{1}, W_{2}, W_{3}\right]^{T}$ and calculate the index weight $\mathrm{W}_{i}$ :

$W_{1}=\bar{W}_{1}\left(\sum_{l=1}^{n} \bar{W}_{1}\right)^{-1}=0.540, W_{2}=\bar{W}_{2} \quad\left(\sum_{l=1}^{n} \bar{W}_{2}\right)^{-1}$ $=0.297, W_{3}=\bar{W}_{3}\left(\sum_{l=1}^{n} \bar{W}_{3}\right)^{-1}=0.163$.

Calculate the maximum eigenvalue of judgment matrix $A,\left|\lambda_{\max }\right|$ :

$$
A \cdot W=\left[\begin{array}{cccc}
a_{11} & a_{12} & \ldots & a_{1 n} \\
a_{21} & a_{22} & \ldots & a_{2 n} \\
\ldots & \ldots & \ldots & \ldots \\
a_{n 1} & a_{n 2} & \ldots & a_{n n}
\end{array}\right]\left[\begin{array}{c}
W_{1} \\
W_{2} \\
\ldots \\
W_{n}
\end{array}\right]
$$

$(A \bullet W)_{1}=1 \times 0.5396+2 \times 0.2970+3 \times 0.1634=1.624$. Similarly, $(A \bullet W)_{2}=0.894,(A \bullet W)_{3}=0.4922$. The last step is consistency testing. When $n=3, R_{1}=0.58$, the judgment matrix has satisfactory consistency. Therefore, the weight of $U 1, U 2$, and $U 3$ is [0.5396 0.29700 .1634$]$. Other index weights can be calculated according to the above method, and the calculation results are shown in Table 1.

When $C_{n}=0.0048, R_{n}=0.90$, and $C_{R 1}=0.0054$, the judgment matrix has satisfactory consistency. When
$\mathrm{C}_{I 2}=0.0192, \mathrm{R}_{I 2}=0.58$, and $C_{R 2}=0.0562$, the judgment matrix has satisfactory consistency. When $C_{I 3}=0.0268$, $\mathrm{R}_{I 3}=0.58$, and $C_{R 3}=0.0562$, the judgment matrix has satisfactory consistency. The weight distribution data of indicators at each level are summarized in Table 2.

\subsection{Intelligent Detection Model of Financial Data. Time} series data is a data column recorded by the same unified indicator in chronological order. All data in the same data column must be of the same caliber and must be comparable. As IOT brings a large amount of time series data, we need to time slice the time series data. The traditional time-series data processing methods include median, extreme value, deviation, variance, year-on-year, month-on-month, and periodic methods. These methods can only roughly summarize the data and form a preliminary understanding. The overlapping slicing method of sliding window is used in this paper. This method sorts and counts the target data according to the time sequence, delimits the length and size of each window, summarizes and calculates the characteristics of each time period window, analyses different data with the same dimension in continuous time periods, and obtains the change trend of the target data. In this paper, it will be solved by convolution neural network, as shown in Figure 4.

Firstly, after the training data is processed by zero mean, principal component analysis is carried out to reduce the input parameters of convolutional neural network model and reduce the correlation between input factors. Then, the new sample dataset obtained by principal component analysis is used for convolutional neural network model learning, and the parameters in convolutional neural network are continuously adjusted by gradient descent. Finally, the test sample data are applied to the model to verify the prediction accuracy of the model.

Since this study only considers the impact of historical data on future enterprise finance, a one-dimensional convolutional neural network is adopted. The model includes two convolutional subnet works, as shown in Figure 5. The input layer is $m k$-dimensional index data output from formula (4), and the output layer is two classifiers. The convolution layer is used to extract different features of the input layer, the linear rectification layer is used to activate neurons in the network according to the linear function, the pooling layer is used to reduce the data dimension, and the full connection layer is used to combine all local features and calculate the final classification result.

In this study, the input data for one-dimensional convolutional neural network is the k-dimensional orthogonal feature $Y_{i j}^{\prime}$ of $M$ companies. Then, three convolutional subnetworks are used to deeply learn the orthogonal feature data of enterprises. The first convolutional network selects 128 convolutional cores with the size of $1 * 3$, and the second convolutional network selects 128 convolutional cores with the size of $1 * 4$. The third subconvolution network selects 128 convolution cores with size of $1 * 5$. The calculation formula is as follows: 


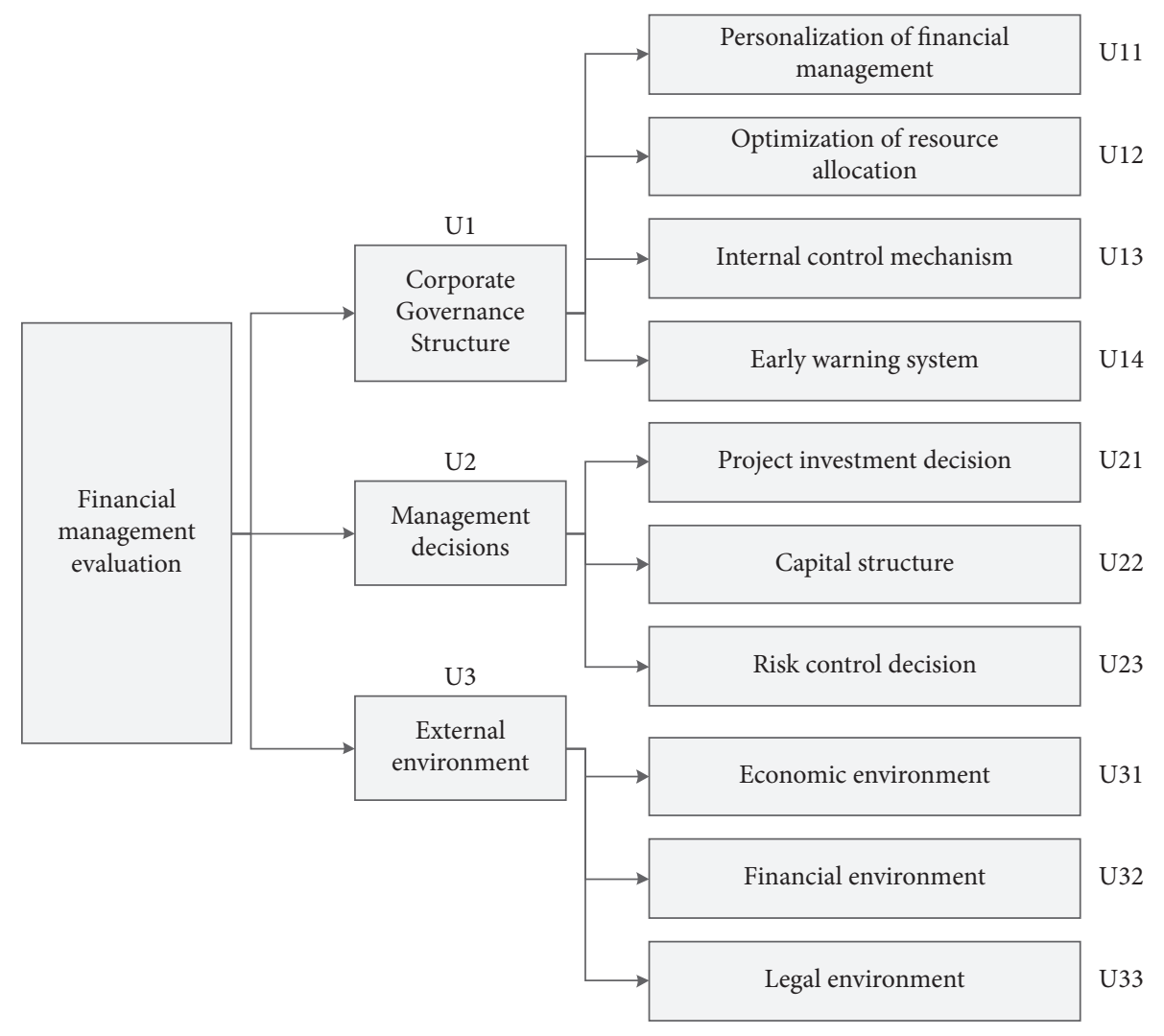

Figure 3: Comprehensive financial management evaluation system.

TABle 1: Index weight.

\begin{tabular}{cccccc}
\hline & U11 & U12 & U13 & U14 & Weight \\
\hline$U 11$ & 1 & 2 & 3 & 4 & 0.6532 \\
$U 12$ & $1 / 2$ & 1 & 2 & 3 & 0.3841 \\
$U 13$ & $1 / 3$ & $1 / 2$ & 1 & 3 & 0.2890 \\
$U 14$ & $1 / 4$ & $1 / 3$ & $1 / 2$ & 1 & 0.1206 \\
\hline
\end{tabular}

TABLe 2: Weight distribution data.

\begin{tabular}{lccccccc}
\hline Index & $U 1$ & $U 2$ & $U 3$ & $U 11$ & $U 12$ & $U 13$ & $U 14$ \\
\hline Weight & 0.5396 & 0.2970 & 0.1634 & 0.4832 & 0.2717 & 0.1569 & 0.0882 \\
\hline Index & $U 21$ & $U 22$ & $U 23$ & $U 31$ & $U 32$ & $U 33$ & \\
\hline Weight & 0.6369 & 0.2583 & 0.1048 & 0.5278 & 0.3325 & 0.1397 & \\
\hline
\end{tabular}

$$
X^{(l)}=f\left(W^{(l)} \cdot X^{(l-1)}+b^{(l)}\right)
$$

where $X^{(l)}$ and $X^{(l-1)}$ are the neuron output values of layer $l$ and layer $l-1, W^{l}$ is convolution kernel, and $b$ is offset. The activation function adopts a modified linear unit. For the input $x$, the weight vector is $\omega$, and the output with offset $b$ is $\max (0, \omega x+b)$. The linear activation function simply sets the threshold to zero, which greatly reduces the computational overhead. Moreover, compared with the expensive operations (exponents, etc.) of sigmoid and tanh neurons, relu can be activated through a simple zero

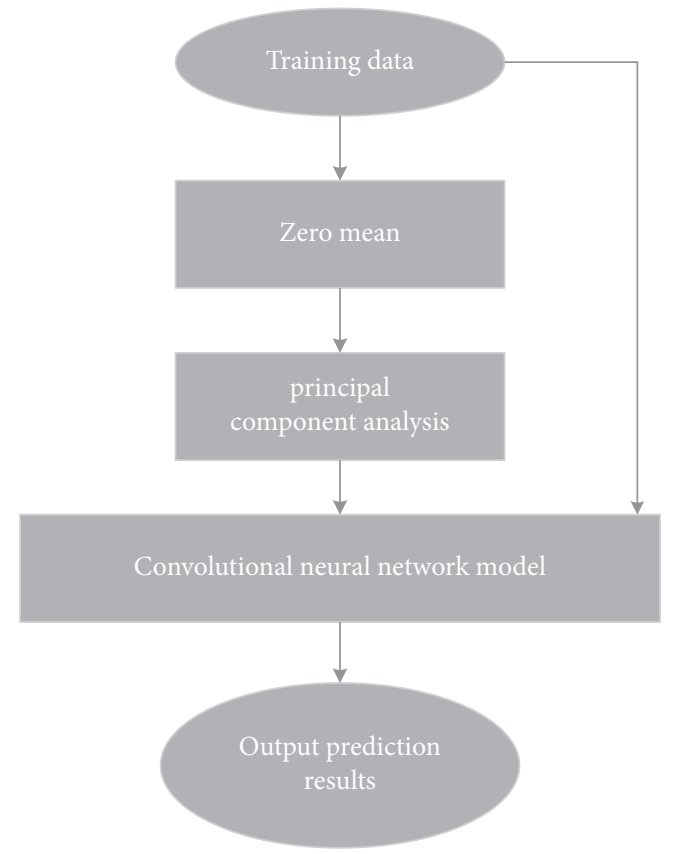

FIgURE 4: Financial management model framework based on convolutional neural network.

threshold matrix and is not affected by saturation. Meanwhile, $L 2$ norm is used to normalize the fitting cost, as shown in 

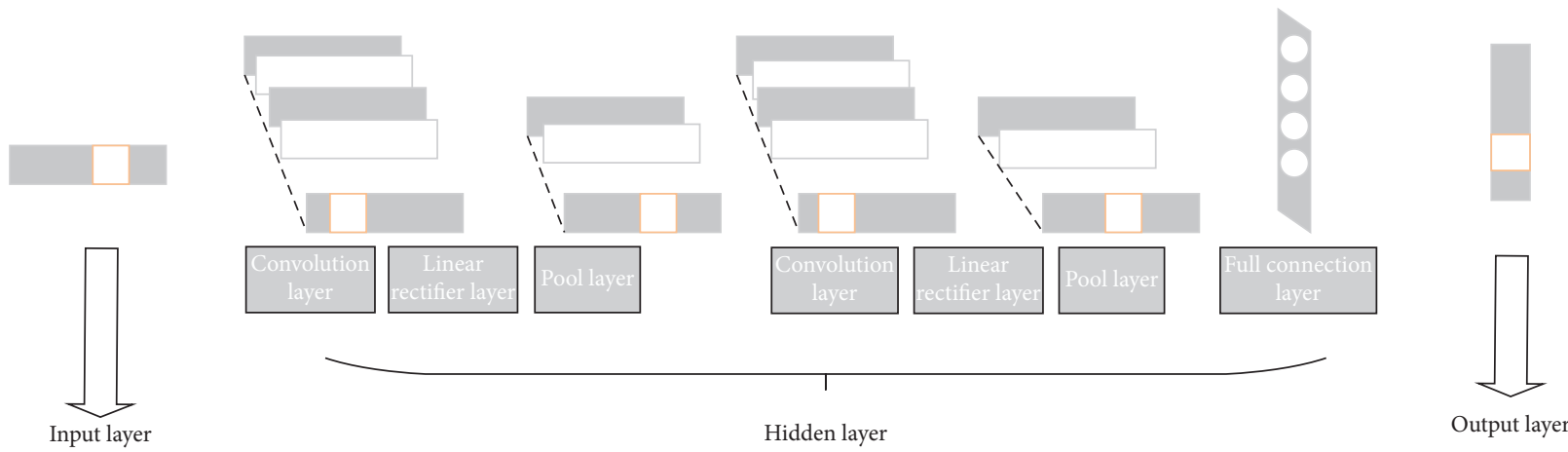

Figure 5: Framework of one-dimensional convolutional neural network model.

$$
C=-n \sum_{1}^{x_{j}}\left[y_{i} \ln a_{j}^{l}+\left(1-y_{i}\right) \ln \left(1-y_{i}\right)\right]+n \sum \lambda \omega^{2}
$$

where the first term represents the cross entropy cost, the second term is the sum of squares of all weights added, and then the factor used $\lambda / 2 n$ to make quantitative adjustment, and $\lambda>0$ is called the normalization parameter. The third convolution subnetwork outputs to the full connection layer and then outputs the final binary result; that is, whether the enterprise is ST in this study, the output result of ST enterprise is 0 and that of non-ST enterprise is 1 [21]. At the same time, this study uses the maximum pooling method to pool local feelings and selects the flexible maximum method to solve the problem of slow learning.

\section{Results and Safety Analysis}

4.1. Data and Empirical Design. In the future, A-share may form a two-way benign expansion of supply and demand, and the regulatory authorities' policies on its stock market are also more effective and in place, which is conducive to the dynamic balance of supply and demand. There are ST system and * ST system in stock market. From the perspective of data availability and effectiveness, it is a reasonable method to use enterprise stock ST or ${ }^{*}$ ST as the symbol of enterprise financial crisis.

This paper first selects the companies that are ST and * ST (hereinafter referred to as ST companies) and then finds out the corresponding companies of each ST or * ST company (hereinafter referred to as non-ST companies) in the companies with normal financial conditions according to the industry and average total assets. Use the financial index data of ST companies and non-ST companies in the previous years of 2016 to predict whether there will be a financial crisis in 2016 (by ST or * ST), compare with the actual situation, count the accuracy of the prediction, and conduct empirical analysis. This paper selects a total of 3513 companies. The reason for data normalization is that the measurement units of each data are different, and the processed data will be between 0 and 1 . If the data is not normalized, the gradient descent is carried out in one unit, so its descent step in each direction is the same. Nonstandardized data will cause the gradient to follow a zigzag route in the direction perpendicular to the contour line when the gradient decreases, which will make the iteration very slow. In general, normalization can make the order of magnitude of each stock index correspond to the length of gradient decline [21].

This paper has conducted four empirical analyses, and the selection of data quantity is shown in Table 3. This paper has conducted four empirical analyses, and the selection of data volume is shown in Table 3. Taking the data of the first few years of 2016 as the training set and the data of the next few years of 2016 as the prediction set, the output result of no financial crisis is 0 and the output result of financial crisis is 1. The judgment result is recorded as $x$, the actual situation of the company is recorded as $y, x$ and $y$ are 0 or 1 , the number of companies in the prediction set is $n$, and the calculation formula of accuracy $P_{a}$ is

$$
P_{a}=\left(1-N^{-1}|X-Y|\right) \times 100 \% .
$$

4.2. Outcome Evaluation Criteria. Because each simulation will randomly take an initial value, the results of each simulation may be different. The experiments were conducted in four groups based on the size of the years of data selected. In general, the nodes in the hidden layers have an impact on the prediction results. If the number of hidden layer nodes is too small, the network cannot have the necessary learning ability and information processing ability. If too much, it will not only increase the complexity of the network structure and make the network more likely to fall into local minima in the learning process but also make the learning speed of the network very slow. The neural network structure has two hidden layers, and the number of them can be determined by the following formula:

$$
\begin{aligned}
L & =\alpha^{2}+(m+n)^{1 / 2} \\
& =\log _{2} m,
\end{aligned}
$$

where $m$ and $n$ represent the nodes of the output layer and the input layer, respectively, $\alpha$ can be any value between 1 and 10 . These methods can only obtain feasible initial values for the nodes of the hidden layer, and this number usually needs to be corrected during training and learning. Generally, two methods of gradually increasing and gradually decreasing are used to correct the number of nodes in the 
TABLE 3: Empirical quantity statistics.

\begin{tabular}{|c|c|c|c|c|c|c|c|}
\hline & & & & No & & & \\
\hline & & $\begin{array}{l}\text { Number of } \\
\text { companies }\end{array}$ & $\begin{array}{c}\text { Amount of } \\
\text { data }\end{array}$ & $\begin{array}{l}\text { Number of } \\
\text { companies }\end{array}$ & $\begin{array}{c}\text { Amount of } \\
\text { data }\end{array}$ & $\begin{array}{l}\text { Number of } \\
\text { companies }\end{array}$ & $\begin{array}{c}\text { Amount of } \\
\text { data }\end{array}$ \\
\hline & & 55 & 2805 & 55 & 2805 & 110 & 5610 \\
\hline 2 years & Total of training set & 55 & 2805 & 55 & 2805 & 110 & 5610 \\
\hline & & 110 & 5610 & 110 & 5610 & 220 & 11220 \\
\hline & Totol of training set & 51 & 5151 & 51 & 5151 & 102 & 10302 \\
\hline 4 years & lotal of training set & 51 & 5151 & 51 & 5151 & 102 & 10302 \\
\hline & & 102 & 10302 & 102 & 10302 & 10506 & 20604 \\
\hline & & 26 & 4576 & 26 & 4576 & 52 & 9152 \\
\hline 8 years & Total of training set & 26 & 4576 & 26 & 4576 & 52 & 9152 \\
\hline & & 52 & 9152 & 52 & 9152 & 104 & 18304 \\
\hline & & 17 & 3842 & 17 & 3842 & 34 & 7684 \\
\hline 12 & Total of training set & 17 & 3842 & 17 & 3842 & 34 & 7684 \\
\hline years & & 34 & 7684 & 34 & 7684 & 68 & 15368 \\
\hline
\end{tabular}

hidden layer to achieve the expected value and reduce the error to a reasonable range. This study uses formula (9) to calculate the hidden layer nodes and finally obtains the nodes of each network, as shown in Table 4.

From the results shown in Figure 6, the accuracy of the model in predicting whether an enterprise will have crisis can be maintained at more than $81 \%$, which shows that the model based on deep learning has a general prediction effect for A-share listed companies, the more the years of data used for prediction, the higher the accuracy of prediction.

In order to test the effect of model training, this study inputs the test data into the model after the above training and observes the accuracy of the test data. From Figure 7, it can be seen that after training, the accuracy of the model on the training set is $79.1 \%$, and the accuracy on the test set is $91.34 \%$. The results show that the accuracy of the test set is slightly higher than that of the training set, which proves that the model has better generalization ability.

\subsection{Intelligent Analysis of Financial Data Based on Deep} Learning. In the pretraining stage, each layer of RBM network is trained separately and unsupervised to ensure that the feature vectors are mapped to different feature spaces and retain the feature information as much as possible. It is pretrained by an unsupervised greedy layer-by-layer method to obtain the weight. In this process, the data is input to the visible layer to generate a vector $V$, which is transmitted to the hidden layer through the weight $W$ to obtain $H$. In the last layer of DBN, a BP network is set up to receive the output eigenvector of RBM as its input eigenvector and train the entity relationship classifier supervised. Moreover, each layer of RBM network can only ensure that the weight in its own layer is optimal for the eigenvector mapping of that layer, not for the eigenvector mapping of the whole DBN. Therefore, the backpropagation network also propagates the error information from top to bottom to each RBM layer and fine-tune the whole DBN network. The process of RBM network training model can be regarded as the initialization of the weight parameters of a deep BP network. The naive Bayesian classifier in this paper is realized by MATLAB programming. In this section, the financial data of traditional indicators are
TABle 4: Network node setup.

\begin{tabular}{lcccc}
\hline & 2 years & 4 years & 8 years & 12 years \\
\hline First hidden layer & 8 & 14 & 29 & 32 \\
Second hidden layer & 4 & 9 & 10 & 10 \\
\hline
\end{tabular}

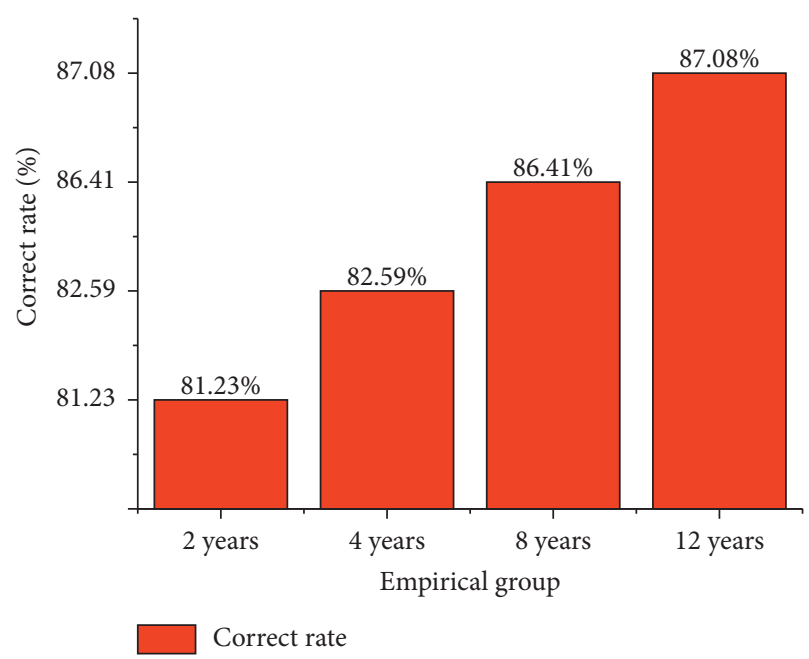

FIGURE 6: Empirical results.

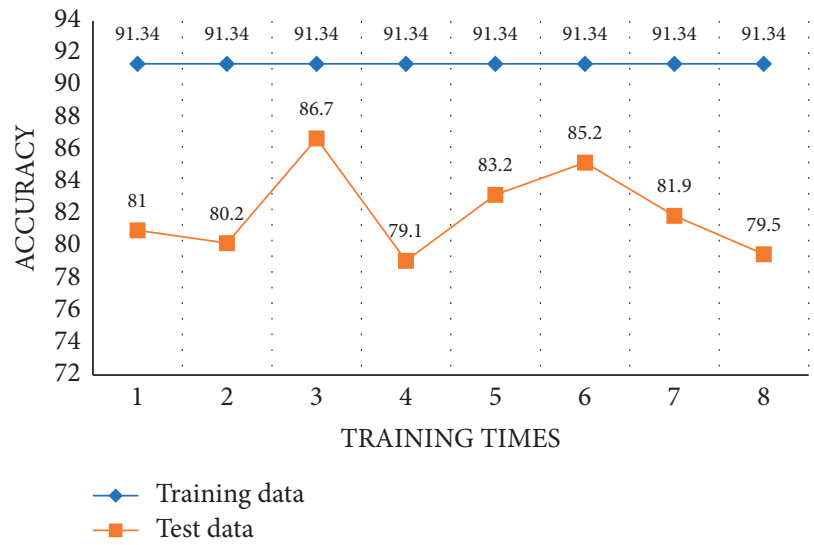

FIgURE 7: The accuracy of training and testing datasets changes with the learning cycle. 


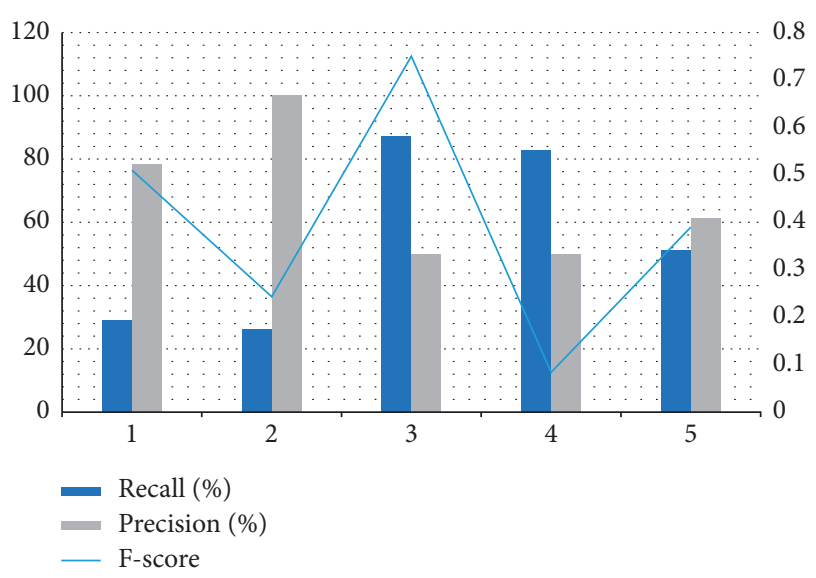

FIGURE 8: Comparison diagram of various forms of model identification. (1) Traditional model. (2) Timing standard in the form of difference. (3) Time series standards in ratio form. (4) The first relative value form of the timing standard. (5) The second relative value form of the timing standard.

processed by the time-series construction method proposed above and then input into the classifier. After running, the classification effects of traditional models and various forms of models can be obtained. The operation results of each model are shown in Figure 8.

In Figure 8, the recall rate of the time series index model in the ratio form and the first relative value form is the highest. In terms of precision, the time series index model in the form of difference is the best. It can also be seen that the recall rate and precision rate show the law of one change and the other. The comparison of classification accuracy of DCNN under different hidden layer structures is shown in Figure 9.

According to Figure 10, hidden levels 1, 2, and 3 show good classification accuracy, all reaching more than $91 \%$. After 600 iterations, the classification accuracy of the second level reaches $98.57 \%$, which is the maximum of the classification accuracy. Therefore, the convolutional neural network model with 3-layer hidden layer structure has good classification accuracy.

In order to further prove the effectiveness and superiority of convolutional neural network model, this work compares it with traditional classical machine learning early warning methods. The methods used for comparison include $k$-nearest neighbor (KNN), support vector machine Gaussian kernel (SVM-RBF), support vector machine linear kernel (SVM linear). The basic parameter setting of the test is the same as the above. The number of neighbors in $\mathrm{KNN}$ is set to 6 and the kernel function parameter of support vector machine is set to 10. Making the weight smaller and smaller, then its corresponding loss function will be smaller and smaller, finally achieving our goal. The smaller the value of the loss function, the more accurate the prediction is. The prediction accuracy of the model established in this study can reach $81.65 \%$ showing a good financial prediction effect. This is because convolutional neural network can better learn the correlation between various indexes and extract the most effective abstract features, so as to ensure the accuracy of prediction results.

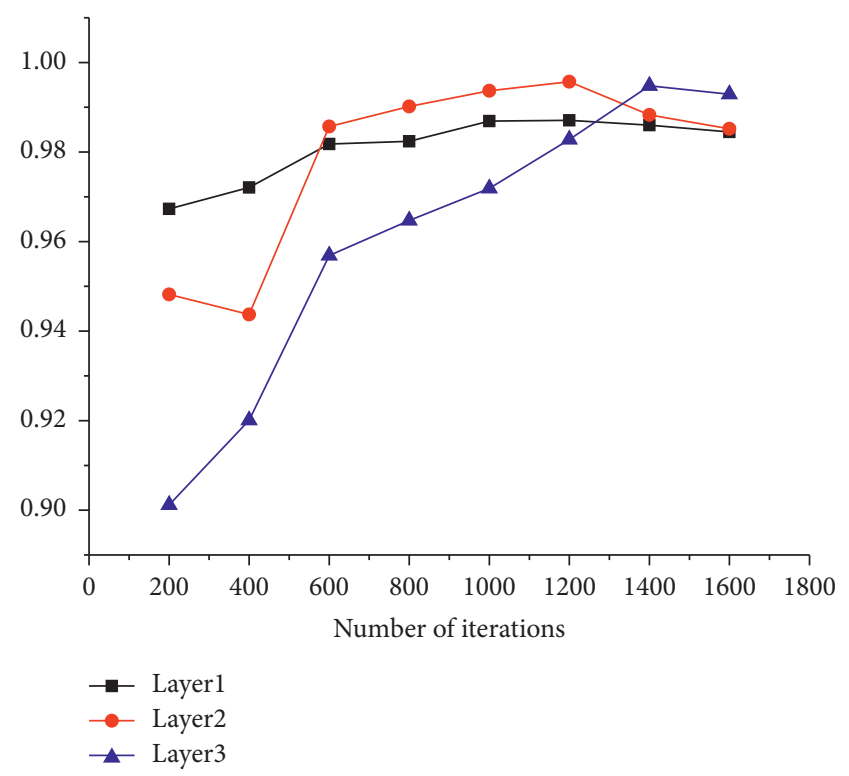

FIGURE 9: DCNN classification accuracy of different hidden layers.

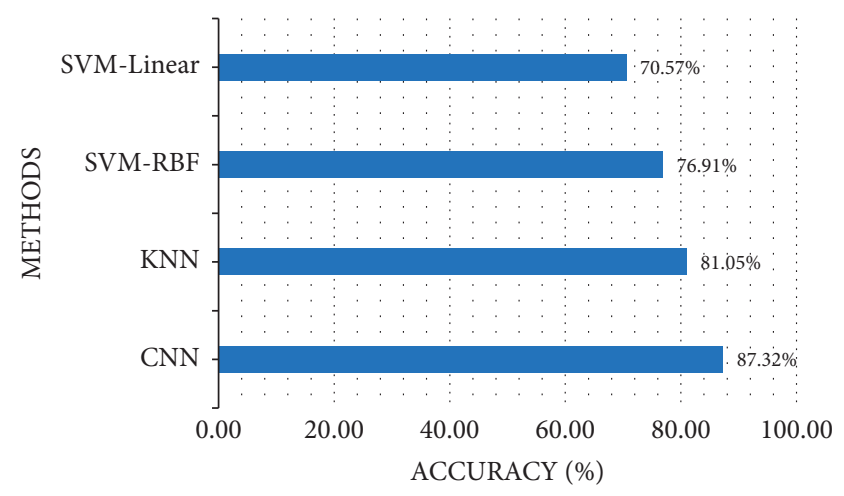

FIgURE 10: Comparison of prediction accuracy of different methods.

\section{Conclusion}

In order to improve the accuracy of corporate financial management evaluation, this paper constructs a financial management evaluation model based on deep learning and uses the data of A-share listed companies from 2007 to 2020 to explore whether deep learning can build a generally applicable financial management model for listed companies. By using the data of different years, it is also concluded that the more the data years the model constructed in this paper uses, the higher the prediction accuracy. The test results show that the intelligent analysis of financial abnormal data based on deep learning is also effective and accurate. Finally, the effectiveness and practicability of the intelligent analysis method are proved by an example.

\section{Data Availability}

The data used to support the findings of this study are included within the article. 


\section{Conflicts of Interest}

All the authors do not have any possible conflicts of interest.

\section{Acknowledgments}

This work was supported by Key R \& D Plan of Shandong Province (Grant no. 2020RKB01367) and the National Social Science Foundation (Grant no. 20BJY033).

\section{References}

[1] O. Tatari, D. C. Lacouture, and M. J. Skibniewski, "Performance evaluation of construction enterprise resource planning systems," Journal of Management in Engineering, vol. 24, no. 4, pp. 198-206, 2008.

[2] B. V. Samorodov, O. O. Sosnovska, and M. O. Zhytar, "Methodical approach to the quantification of enterprise financial security level," Financial and credit activity: Problems of Theory and Practice, vol. 1, no. 32, pp. 269-277, 2020.

[3] R. Zajarskas and J. Ruževičius, "Evaluation of the effectiveness of the quality management system of the service enterprise," Ekonomika ir vadyba, no. 15, pp. 857-864, 2010.

[4] С. В. Бословяк, "Algorithm OF complex evaluation OF enterprise investment enterprise," TIME DESCRIPTION OF ECONOMIC REFORMS, no. 1, pp. 35-43, 2018.

[5] V. Levytskyi, "The optimization of system financial management of enterprise based on the analysis of investments in its marketing activities," Economic journal of Lesya Ukrainka Volyn National University, vol. 2, no. 18, pp. 101-108, 2019.

[6] S. Mekadmi and R. Louati, "An evaluation model of user satisfaction with enterprise resource planning systems," Electronic Journal of Information Systems Evaluation, vol. 21, no. 2, pp. 143-157, 2018.

[7] A. Meylis, "Analysis and prevention of enterprise financial risk under the new tax policy," Open Journal of Business and Management, vol. 7, no. 4, pp. 1943-1952, 2019.

[8] P. S. Rosa and I. R. Gartner, "Financial distress in Brazilian banks: an early warning model," Revista Contabilidade \& Finanças, vol. 29, no. 77, pp. 312-331, 2018.

[9] G. S. Ng, C. Quek, and H. Jiang, "FCMAC-EWS: a bank failure early warning system based on a novel localized pattern learning and semantically associative fuzzy neural network," Expert Systems with Applications, vol. 34, no. 2, pp. 989-1003, 2008.

[10] Z. Wu and W. Chu, "Sampling strategy analysis of machine learning models for energy consumption prediction," in Proceedings of the 2021 IEEE 9th International Conference on Smart Energy Grid Engineering (SEGE), pp. 77-81, IEEE, Oshawa, ON, Canada, Augugust 2021.

[11] M. Zhao, A. Jha, Q. Liu et al., "Faster Mean-shift: GPUaccelerated clustering for cosine embedding-based cell segmentation and tracking," Medical Image Analysis, vol. 71, Article ID 102048, 2021.

[12] M. D. C. H. Sundaram, A. John, and D. D. Seligmann, "Can blog communication dynamics be correlated with stock market activity?" Journal of Machine Learning Research, vol. 11, no. 9, pp. 89-93, 2008.

[13] P. C. Tetlock, M. S. Tsechansky, and S. Macskassy, "More than words: quantifying language to measure firms' fundamentals," The Journal of Finance, vol. 63, no. 3, pp. 1437-1467, 2008.

[14] M. M. Najafabadi, F. Villanustre, T. M. Khoshgoftaar, N. Seliya, R. Wald, and E. A. Muharemagic, "Deep learning applications and challenges in big data analytics," Journal of big data, vol. 2, no. 1, pp. 1-21, 2015.

[15] A. L. Jones, "Have internet message boards changed market behavior?" Info, vol. 8, no. 5, pp. 67-76, 2006.

[16] N. Yudistira and T. Kurita, "Gated spatio and temporal convolutional neural network for activity recognition: towards gated multimodal deep learning," EURASIP Journal on Image and Video Processing, vol. 2017, no. 1, pp. 1-12, 2017.

[17] M. M. Hassan, M. G. R. Alam, M. Z. Uddin, and S. Huda, "Human emotion recognition using deep belief network architecture," Information Fusion, vol. 51, pp. 10-18, 2019.

[18] S. Pirmoradi, M. Teshnehlab, N. Zarghami, and S. Arash, "The self-organizing restricted Boltzmann machine for deep representation with the application on classification problems," Expert Systems with Applications, vol. 149, Article ID 113286, 2020.

[19] P. Save, P. Tiwarekar, K. N. Jain, and M. Neha, "A novel idea for credit card fraud detection using decision tree," International Journal of Computer Applications, vol. 161, no. 13, pp. 6-9, 2017.

[20] Y. Bai, C. Gu, Q. Chen, J. Xiao, D. Liu, and S. Tang, "The challenges that head nurses confront on financial management today: a qualitative study," International journal of nursing sciences, vol. 4, no. 2, pp. 122-127, 2017.

[21] S. Gupta, T. Gupta, and G. Shainesh, "Navigating from programme loyalty to company loyalty," IIMB management review, vol. 30, no. 3, pp. 196-206, 2018. 